\title{
Periodic System of Atoms in Biquaternionic Representation
}

\author{
Lyudmila Alexeyeva \\ Institute of Mathematics and Mathematical Modeling, Almaty, Kazakhstan \\ Email: alexeeva@math.kz
}

How to cite this paper: Alexeyeva, L. (2018) Periodic System of Atoms in Biquaternionic Representation. Journal of Modern Physics, 9, 1633-1644. https://doi.org/10.4236/jmp.2018.98102

Received: June 6, 2018

Accepted: July 21, 2018

Published: July 24, 2018

Copyright $\odot 2018$ by author and Scientific Research Publishing Inc. This work is licensed under the Creative Commons Attribution International License (CC BY 4.0).

http://creativecommons.org/licenses/by/4.0/

\section{(c) (i) Open Access}

\begin{abstract}
Private monochromatic solutions of the free-field equation of electro-gravimagnetic charges and currents are constructed in the differential algebra of biquaternions, which describe elementary particles as standing electro-gravimagnetic waves. The two classes of solutions of this biquaternionic wave equation have been investigated, generated by scalar potentials (pulsars) and vectorial potentials (spinors). Their asymptotic properties are considered, on the base of which they are classified into heavy (boson) and light (lepton) elementary particles. The biquaternion representation of the hydrogen atom is given. The periodic system of elements is produced, which is built on the principle of the musical structure of a simple gamma.
\end{abstract}

\section{Keywords}

Biquaternion, Elementary Particle, Frequency, Standing EGM-Wave, Pulsar, Spinor, Boson, Lepton, Atom, Hydrogen, Periodic System, Musical Scale

\section{Introduction}

In [1] [2] [3], the author developed a biquaternion model of the electrogravimagnetic field (EGM-field) and electro-gravimagnetic interactions. Its basis is made up of biquaternion representations of the generalized Maxwell and Dirac equations. The biquaternion representation of the Maxwell equations expresses the biquaternion of the mass-charge density and the EGM-current through the bigradient of the EGM-field tension. The biquaternionic representation of Dirac equations determines the transformation of the density of mass charges and currents under the influence of external EGM-fields. In particular, in the absence of external fields, it is the biquaternionic wave (biwave) equation for the free field of mass-charges and currents, which is a field analogue of first Newton's law (inertia law). 
Here we construct particular monochromatic solutions of this equation that describe elementary particles as standing EGM-waves. They can be divided into two class, generated by scalar potentials (pulsars) and vector ones (spinors). Their asymptotic properties are investigated, on the basis of which they are classified into heavy (bosons) and light (leptons) elementary particles. It is shown that bosons are spherical harmonic pulsars whose mass-charge density is determined by their frequency of oscillations. This allows us to build periodic systems of elementary particles based on the classical harmonic musical scale.

In particular, the biquaternion representation of the hydrogen atom is given and the periodic system is produced, which is built on the principle of the musical structure of a simple harmonic scale.

\section{Inertia Law for Monochromatic Fields of Charges-Currents}

The equation for the free field of charge-currents has the form of a homogeneous biwave equation [1] [2]:

$$
\nabla^{-} \Theta(\tau, x) \triangleq\left(\partial_{\tau}-i \nabla\right) \circ(i \rho(\tau, x)+J(\tau, x))=0
$$

Here, $\Theta(\tau, x)$ is the biquaternion of the charge-current, the secular part of which $\rho(\tau, x)$ describes the density of the electric and gravimagnetic charge (EGM-charge or mass-charge), and the vector $J(\tau, x)$ is the density of the electric and gravimagnetic current (EGM-current), more exactly:

$$
\begin{gathered}
\rho=\frac{1}{\sqrt{\varepsilon}} \rho^{E}-\frac{i}{\sqrt{\mu}} \rho^{H}, \\
J=\sqrt{\mu} j^{E}-i \sqrt{\varepsilon} j^{H},
\end{gathered}
$$

where $\rho^{E}(x, t), j^{E}(x, t)$ are the electric charge and electric current densities, $\rho^{H}(x, t), j^{H}(x, t)$ are the gravimagnetic charge density and current density; $\varepsilon, \mu$ are the constants of electric conductivity and magnetic permeability of vacuum, $c=1 / \sqrt{\varepsilon \mu}$ is speed of light, $i$ is imaginary unit.

The action of the biquaternion differential operators $\nabla^{-}$and $\nabla^{+}$(mutual bigradients) is determined, according to the quaternion multiplication rule (see Appendix), by the formula

$$
\begin{aligned}
\nabla^{ \pm} \mathrm{F}(\tau, x) & =\left(\partial_{\tau} \pm i \nabla\right) \circ(f(\tau, x)+F(\tau, x)) \\
& =\left(\partial_{\tau} f \mp i \operatorname{div} F\right)+\left\{ \pm i \operatorname{grad} f+\partial_{\tau} F \pm i \operatorname{rot} F\right\}
\end{aligned}
$$

The biquaternion of the energy-momentum of the F-field is given by

$$
\Sigma(\tau, x)=W(\tau, x)+i P(\tau, x) \triangleq 0.5 \mathrm{~F} \circ \mathrm{F}^{*}
$$

where $\mathrm{F}^{*}$ is the conjugate biquaternion

$$
\mathrm{F}^{*} \triangleq \bar{f}(\tau, x)-\bar{F}(\tau, x)
$$

Here the bar over the symbol means complex conjugation.

The scalar part $W$ is the energy density of the F-field, and $P$ is the analog of the generalized Poynting vector of the F-field, just as a generalized Poynting 
vector is constructed in the biquaternionic representation of the electromagnetic field (in detail about the differential algebra of biquaternions with an application in electrodynamics, see [4]).

The scalar part of the Equation (1) is the law of conservation of the EGM-charge:

$$
\partial_{\tau} \rho+\operatorname{div} J=0
$$

and the vectorial part describes the relationship between charges and currents in the absence of external EGM-fields:

$$
\partial_{\tau} J-i \operatorname{rot} J+\operatorname{grad} \rho=0 .
$$

For monochromatic fields of frequency $\omega$, the biquaternion of the charge-current can be represented in the form

$$
\Theta(\tau, x) \triangleq \Theta(x, \omega) \exp (i \omega \tau), \quad \omega>0 .
$$

In this case, from the Equation (2) we obtain the equation for biquaternions of complex amplitudes ( biamplitudes) $\Theta(x, \omega)$ :

$$
(\omega-\nabla) \circ(i \rho(x)+J(x))=0 .
$$

As

$$
(\omega+\nabla) \circ(\omega-\nabla)=(\omega-\nabla) \circ(\omega+\nabla)=\omega^{2}+\Delta,
$$

it follows that the biamplitudes satisfy the Helmholtz equation

$$
\Delta \Theta+\omega^{2} \Theta=0
$$

and monochromatic solutions have the form:

$$
\Theta(\tau, x)=\exp (i \omega \tau)(\omega+\nabla) \circ\left(\psi^{0}(x, \omega)+\sum_{j=1}^{3} \psi^{j}(x, \omega) e_{j}\right),
$$

where the potentials $\psi^{j}$ are arbitrary solutions of the homogeneous Helmholtz equation

$$
\Delta \psi+\omega^{2} \psi=0
$$

which have the form of a surface integral

$$
\psi^{j}(x, \omega)=\int_{\|\xi\|=\omega} \varphi^{j}(\xi, \omega) \mathrm{e}^{-i(\xi, x)} \mathrm{d} S(\xi)
$$

for any function $\phi^{j}$ that is integrable on a sphere of radius $\omega$.

\section{Biquaternions of Harmonic Elementary Particles}

We consider particular solutions of the Helmholtz equation [5] [6]

$$
\psi_{n m}(x, \omega)=j_{n}(\omega r) Y_{n}^{m}(\vartheta, \phi)
$$

where $j_{n}(\omega r)$ are spherical Bessel functions of order $n=0,1,2, \cdots ; Y_{n}^{m}(\vartheta, \phi)$ spherical harmonics of order $(n, m), m=0,1,2, \cdots$

$$
Y_{n}^{m}(\vartheta, \phi)=P_{n}^{m}(\cos \vartheta) \exp (\operatorname{im} \phi)
$$

$P_{n}^{m}(\ldots)$ are the associated Legendre polynomials, $(r, \vartheta, \phi)$ are spherical 
coordinates.

It is natural to take these solutions for the construction of elementary particles, which can be called harmonic. Among them we select the ones generated by the scalar potential, which we call pulsars.

$$
\begin{aligned}
\Theta_{n m}^{0}(x, \omega) & =(\omega+\nabla) \circ \psi_{n m}(x, \omega) \\
& =\omega \psi_{n m}(x, \omega)+\operatorname{grad} \psi_{n m}(x, \omega)
\end{aligned}
$$

and particles, generated by a vector potential, we call spinors.

$$
\begin{aligned}
& \Theta_{n m}^{j}(x, \omega)=(\omega+\nabla) \circ \psi_{n m}(x, \omega) e_{j} \\
& =-\operatorname{div}\left(\psi_{n m}(x, \omega) e_{j}\right)+\left\{\omega \psi_{n m}(x, \omega) e_{j}+\operatorname{rot}\left(\psi_{n m}(x, \omega) e_{j}\right)\right\}
\end{aligned}
$$

The latter are polarized in the direction of the coordinate axes, respectively, to the index $j=1,2,3$.

\section{Biquaternions of Monochromatic Structures. Crystals}

Using structural biquaternions of arbitrary form $\mathrm{K}(x)$, on their basis, by the operation of biquaternion convolution

$$
\begin{aligned}
& \Theta(x, \omega) * \mathrm{~K}(x)=(i \rho+J) *(k+K) \\
& =\left\{i \rho * k-\sum_{j=1}^{3}\left(J_{j} * K_{j}\right)\right\}+\left\{i \rho * K+J * k+\sum_{j, l, m=1}^{3} \varepsilon_{j l m}\left(J_{j} * K_{l}\right) e_{m}\right\}
\end{aligned}
$$

where $\varepsilon_{j l m}$ is the Levi-Civita pseudo-tensor, it is possible to construct a variety of monochromatic fields of charge-currents:

$$
\Theta(x, \omega)=\sum_{j=0}^{3} \Theta_{n m}^{j}(x, \omega) * \mathrm{~K}_{j}(x)
$$

In (8) there are functional convolutions, which for integrable functions have the integral form:

$$
\rho(x) * k(x)=\int_{R^{3}} \rho(y) k(x-y) \mathrm{d} y_{1} \mathrm{~d} y_{2} \mathrm{~d} y_{3}
$$

Component convolutions for vectors are written similarly. By virtue of the differentiation property of convolution, the convolutions (9) are also solutions of the equations (1).

Formulas (9) allow us to construct various crystal lattices from harmonic elementary particles, if we take lattices as the structural biquaternion the different shifts of the $\delta$-function, and other generalized functions.

We give here a simple example of an inhomogeneous rectangular lattice with variable step $\left(h_{l}, h_{m}, h_{n}\right)$ and weight $a^{l m n}$ :

$$
\mathrm{K}(x)=\sum_{l=0}^{L} \sum_{m=0}^{M} \sum_{n=0}^{N} a^{l m n} \delta\left(x_{1}-h_{l}\right) \delta\left(x_{2}-h_{m}\right) \delta\left(x_{3}-h_{n}\right)
$$

It corresponds, for example, to such a crystalline $\omega$-pulsar

$$
\Theta(x, \omega)=\sum_{l=0}^{L} \sum_{m=0}^{M} \sum_{n=0}^{N} a^{l m n} \theta^{0}\left(x_{1}-l h_{l}, x_{2}-m h_{m}, x_{3}-n h_{n}, \omega\right)
$$

The formulas (7)-(9) make it possible to construct a wide variety of monochromatic structures, such as crystals, bodies, tissues and filaments (for 
more details, see [2]). And their frequency superpositions are generally immeasurable.

\section{Elementary Spherical Harmonic Pulsars and Their Properties}

Among the solutions of the Helmholtz Equations (7), only one is spherically symmetric [5]. It is

$$
\psi_{00}(x, \omega)=j_{0}(\omega r)=\frac{\sin \omega r}{\omega r}
$$

Here $r=\|x\|=\sqrt{x_{1}^{2}+x_{2}^{2}+x_{3}^{2}}, \quad j_{0}(\omega r)$ are spherical Bessel functions.

The biamplitude of the corresponding pulsar is

$$
\begin{aligned}
& \Theta^{0}(x, \omega)=(\omega+\nabla) \circ \psi_{00}(x, \omega) \\
& =\omega \frac{\sin \omega r}{\omega r}+\operatorname{grad} \frac{\sin \omega r}{\omega r}=\cdots=\frac{\sin \omega r}{r}+\left(\frac{\cos \omega r}{r}-\frac{\sin \omega r}{\omega r^{2}}\right) e_{x}, e_{x}=x / r
\end{aligned}
$$

Whence follows

$$
\begin{gathered}
i \rho_{1}^{0}+J_{1}^{0}=r^{-1}\left\{\sin \omega r+\left(\cos \omega r-\frac{\sin \omega r}{\omega r}\right) e_{x}\right\} \mathrm{e}^{i \omega \tau} \\
\rho^{0}=-\frac{i \sin \omega r}{r} \mathrm{e}^{i \omega \tau}, \quad J^{0}=\left(\frac{\cos \omega r}{r}-\frac{\sin \omega r}{\omega r^{2}}\right) \mathrm{e}^{-i \omega \tau} e_{x} \\
\Rightarrow\left|\rho^{0}\right|=\frac{|\sin \omega r|}{r}, \quad\left\|J^{0}\right\|=\left|\frac{\cos \omega r}{r}-\frac{\sin \omega r}{\omega r^{2}}\right|
\end{gathered}
$$

We denote by

$$
j(z)=\cos z-\frac{\sin z}{z}=-z j_{1}(z) .
$$

Calculating the biquaternion of its energy-momentum

$$
\begin{aligned}
\Xi^{0}(x, \omega)= & W^{0}+i P^{0}=0.5 \Theta^{0} \mathrm{e}^{i \omega \tau} \circ\left(\Theta^{0}\right)^{*} \mathrm{e}^{-i \omega \tau} \\
\triangleq & 0.5 r^{-2}\left(\sin \omega r+j(\omega r) e_{x}\right) \circ\left(\sin \omega r-j(\omega r) e_{x}\right) \\
= & 0.5 r^{-2}\left\{\left(\sin ^{2} \omega r+j^{2}(\omega r)\right)+\sin \omega r j(\omega r) e_{x}\right. \\
& \left.-\sin \omega r j(\omega r) e_{x}-j^{2}(\omega r)\left[e_{x}, e_{x}\right]\right\},
\end{aligned}
$$

we get

$$
W^{0}=0.5 r^{-2}\left(\sin ^{2} \omega r+j^{2}(\omega r)\right), \quad P \equiv 0 .
$$

It follows from (11)-(12) that the density of the mass-charge decreases as $r^{-1}$ by increasing $r$, and the oscillation energy decays even more rapidly, as $r^{-2}$.

It is interesting to investigate the asymptotic of these quantities as $r \rightarrow 0, \omega=$ const. Since

$$
\begin{aligned}
j(\omega r) & =(\omega r)^{2} j_{1}(\omega r)=\cos \omega r-\frac{\sin \omega r}{\omega r} \\
& =1-\frac{(\omega r)^{2}}{2}-1+\frac{(\omega r)^{3}}{6 \omega r}+\cdots \sim-\frac{(\omega r)^{2}}{3} \quad r \rightarrow 0,
\end{aligned}
$$


from (11) and (13) we obtain: by $r \rightarrow 0$

$$
\begin{gathered}
\left|\rho^{0}\right|=\omega+o(\omega r) \sim \omega, \\
\left\|J^{0}\right\| \approx \frac{\omega r+0.5 \omega^{2} r^{2}}{r}-\frac{\omega r-\omega^{3} r^{3} / 6}{\omega r^{2}} \approx 0.5 \omega^{2} r+\omega^{2} r / 6 \sim \frac{2}{3} \omega^{2}, \\
W \sim 0.5\left(\omega^{2}+\omega^{4} r^{2} / 9\right) \sim 0.5 \omega^{2} .
\end{gathered}
$$

Let us now list these properties.

The properties of spherical pulsars. In spherical harmonic pulsars at the center at $x=0$, the mass-charge density is equal to its oscillation frequency $\omega$; the density of the EGM-current is zero; the energy density is equal to $0.5 \omega^{2}$; and the Poynting vector equal to zero everywhere.

Proceeding from these properties of mass-charge density, spherical harmonic pulsars are heavy elementary particles-bosons.

Non-spherical harmonic pulsars (6) for $n>0$ have zero density at $x=0$, because [5]

$$
j_{n}(z)=\frac{z^{n}}{(2 n+1) ! !}(1+o(z)) \quad z \rightarrow 0 .
$$

They are light elementary particles-leptons.

\section{Elementary Spherical Harmonic Spinors and Their Properties}

Consider a spinor polarized in direction $X_{1}$ :

$$
\Theta_{1}^{0}(x, t)=i \rho_{1}^{0}+J_{1}^{0}=\Theta_{1}^{0}(x, \omega) \mathrm{e}^{i \omega \tau},
$$

whose biamplitude is

$$
\begin{aligned}
\Theta_{1}^{0}(x, \omega)=(\omega+\nabla) \circ j_{0}(\omega r) e_{1} \\
=-\operatorname{div}\left(j_{0}(\omega r) e_{1}\right)+\omega j_{0}(\omega r) e_{1}+\operatorname{rot}\left(j_{0}(\omega r) e_{1}\right) \\
=-r,\left(\frac{\cos \omega r}{r}-\frac{\sin \omega r}{\omega r^{2}}\right) \\
+\left\{\frac{\sin \omega r}{r} e_{1}+\left(\frac{\cos \omega r}{r}-\frac{\sin \omega r}{\omega r^{2}}\right)\left(r_{, 3} e_{2}-r_{, 2} e_{3}\right)\right\}, \quad r,,_{j}=\frac{x_{j}}{\|x\|}
\end{aligned}
$$

Whence follows

$$
\begin{gathered}
\rho_{1}^{0}=-\frac{i x_{1}}{r^{2}} j(\omega r), \\
J_{1}^{0}=r^{-1}\left(e_{1} \sin \omega r+j(\omega r)\left(r_{3} e_{2}-r_{,_{2}} e_{3}\right)\right) ; \\
\left|\rho_{1}^{0}\right|=\left|\frac{r_{1}}{r} j(\omega r)\right|, \quad\|J\|=\sqrt{\frac{\sin ^{2} \omega r}{r^{2}}+j^{2}(\omega r) \frac{\left(x_{2}^{2}+x_{3}^{2}\right)}{r^{4}}}
\end{gathered}
$$

We calculate the biquaternion of the energy-momentum and its asymptotic:

$$
\Xi_{1}^{0}(x, \omega)=W_{1}^{0}+i P_{1}^{0},
$$




$$
\begin{aligned}
2 W_{1}^{0}= & \frac{\sin ^{2} \omega r}{r^{2}}+\left(\frac{r,_{1}}{r}\right)^{2} j^{2}(\omega r)+j^{2}(\omega r) \frac{\left(x_{2}^{2}+x_{3}^{2}\right)}{r^{4}} \\
= & \frac{\sin ^{2} \omega r}{r^{2}}+j^{2}(\omega r) \frac{\left(x_{1}^{2}+x_{2}^{2}+x_{3}^{2}\right)}{r^{4}} \\
= & r^{-2}\left(\sin ^{2} \omega r+j^{2}(\omega r)\right) . \\
P_{1}^{0} \equiv 0 . &
\end{aligned}
$$

We construct their asymptotic for $r \rightarrow 0$ with allowance (13):

$$
\begin{gathered}
\left|\rho_{1}^{0}\right| \sim\left|\frac{x_{1}}{r^{2}}\left(1-\frac{\omega^{2} r^{2}}{2}-1+\frac{\omega^{3} r^{3}}{6 \omega r}\right)\right|=\frac{1}{3}\left|x_{1}\right| \omega^{2} \rightarrow 0, \\
\|J\| \sim \sqrt{\frac{(\omega r)^{2}}{r^{2}}+\frac{(\omega r)^{4}}{9} \frac{\left(x_{2}^{2}+x_{3}^{2}\right)}{r^{4}}} \sim \omega \rightarrow \omega, \\
W \sim \frac{1}{2 r^{2}}\left(1-\frac{2 \omega r}{\omega r}+\frac{8(\omega r)^{3}}{6 \omega r}+\frac{\left(\omega r-(\omega r)^{3} / 6\right)^{2}}{\omega^{2} r^{2}}\right) \\
=\frac{1}{2 r^{2}}\left(1-2+\frac{4}{3}(\omega r)^{2}+1-\frac{1}{3}(\omega r)^{2}+\frac{1}{36}(\omega r)^{4}\right) \sim 0.5 \omega^{2} .
\end{gathered}
$$

Following (14), we easily obtain a biquaternion representation of the spherical spinor polarized along the vector $e,\|e\|=1$ :

$$
\begin{aligned}
\Theta_{e}^{0}(x, \omega) & =(\omega+\nabla) \circ j_{0}(\omega r) e \\
& =-\operatorname{div}\left(j_{0}(\omega r) e\right)+\omega j_{0}(\omega r) e+\operatorname{rot}\left(j_{0}(\omega r) e\right) \\
& =i \rho_{e}^{0}+J_{e}^{0}
\end{aligned}
$$

where

$$
\rho_{e}^{0}=-\frac{e_{x}}{r} j(\omega r), \quad J_{e}^{0}=r^{-1}\left(e \sin \omega r+j(\omega r) \varepsilon_{j k l} x_{j} x_{k} e_{l}\right)
$$

with the same asymptotic properties.

So we have the following properties of the constructed spinors.

Properties of harmonic spherical spinors. For spherical harmonic spinors at the center (for $x=0$ ), the mass-charge density is zero, the norm of the density of the EGM-current vector is $\omega$, the energy density is $\omega^{2} / 2$, the Poynting vector is equal to zero.

Thus, spherical harmonic spinors in terms of the density of the EGM-charge belong to light elementary particles-leptons.

\section{Biquaternion Model of the Hydrogen Atom}

Thus, we have shown that among the monochromatic solutions of the chargecurrent free field Equations (1), only harmonic spherical pulsars have a nonzero density at their center, which is not the case for harmonic spinors. This suggests that spherical harmonic pulsars can be used to construct a biquaternion model of atoms. 
The simplest atom is hydrogen $(\mathrm{H})$. The simplest harmonic elementary particle is the above described spherical pulsar. I think they can be identified, namely:

The hydrogen atom is a spherical harmonic pulsar with fixed oscillation frequency $\omega_{H_{0}}$. It has the following biquaternionic representation

$$
\mathrm{H}_{0}(\tau, x)=r^{-1}\left\{\sin \omega_{H_{0}} r+\left(\cos \omega_{H_{0}} r-\frac{\sin \omega_{H_{0}} r}{\omega_{H_{0}} r}\right) e_{x}\right\} \mathrm{e}^{i \omega_{H_{0}} \tau} .
$$

The asymptotic properties of its density at the center of an atom are related to the frequency of the oscillations:

$$
\left|\rho_{H_{0}}(x, \tau)\right| \sim \omega_{H_{0}}, \quad\left\|J_{H_{0}}(x, \tau)\right\| \sim \frac{2}{3} \omega^{2} r, \quad W_{H_{0}}(x) \sim 0.5 \omega^{2}, \quad r \rightarrow 0 .
$$

The nodes of this standing wave with respect to the mass density $\left|\rho_{H_{0}}\right|$ are spheres whose radius is determined by a simple trigonometric equation

$$
\sin \omega_{H_{0}} r_{k}=0 \Rightarrow r_{k}=\frac{\pi k}{\omega_{H_{0}}}, k=1,2, \cdots
$$

To determine the nodes of this standing wave from the energy density $W_{H_{0}}$, it is necessary to find the zeros of the more complicated equation:

$$
\omega_{H_{0}}^{2} r_{k}^{2}+\omega_{H_{0}} r_{k} \sin 2 \omega_{H_{0}} r_{k}-\sin ^{2} \omega_{H_{0}} r_{k}=0
$$

where $r_{k}=\frac{z_{k}}{\omega_{H_{0}}}, z_{k}$ are the roots of the transcendental equation:

$$
f(z)=z^{2}+z \sin 2 z-\sin ^{2} z=0
$$

However, this equation has no real roots.

Using the representation of complex charges and currents through electric and gravimagnetic charges and currents (1), we obtain for the hydrogen atom the following expressions for its electric and gravimagnetic charges, electric and gravimagnetic currents:

$$
\begin{gathered}
\rho_{H_{0}}^{E}(t, x)=-\frac{\sqrt{\varepsilon}}{r} \cos w_{H_{0}} t \sin \frac{w_{H_{0}}\|x\|}{c}, \\
\rho_{H_{0}}^{H}(t, x)=\frac{\sqrt{\mu}}{r} \sin w_{H_{0}} t \sin \frac{w_{H_{0}}\|x\|}{c} \\
J_{H_{0}}^{E}(t, x)=\frac{1}{\sqrt{\mu} r} \cos w_{H_{0}} t\left(\cos \frac{w_{H_{0}}\|x\|}{c}-\frac{c}{w_{H_{0}} r} \sin \frac{w_{H_{0}}\|x\|}{c}\right) e_{x} \\
J_{H_{0}}^{H}(t, x)=-\frac{1}{\sqrt{\varepsilon} r} \sin w_{H_{0}} t\left(\cos \frac{w_{H_{0}}\|x\|}{c}-\frac{c}{w_{H_{0}} r} \sin \frac{w_{H_{0}}\|x\|}{c}\right) e_{x}
\end{gathered}
$$

Accordingly, in the initial space-time, the biquaternion of hydrogen has the form:

$$
\mathrm{H}_{0}(t, x)=\frac{\mathrm{e}^{i w_{H_{0}} t}}{r}\left\{-\sin \left(w_{H_{0}} r / c\right)+\left(\cos \left(w_{H_{0}} r / c\right)-\frac{c \sin \left(w_{H_{0}} r / c\right)}{w_{H_{0}} r}\right) e_{x}\right\} .
$$


Here $e_{x}=x /\|x\|$, vibration frequency of a hydrogen atom $w_{H_{0}}=c \omega_{H_{0}}$. Accordingly (17), by $r \rightarrow 0$

$$
\left|\rho_{H_{0}}(x, \tau)\right| \sim c w_{H_{0}}, \quad\left\|J_{H_{0}}(x, \tau)\right\| \sim \frac{2}{3}\left(c w_{H_{0}}\right)^{2} r, \quad W_{H_{0}}(x) \sim \frac{\left(c w_{H_{0}}\right)^{2}}{2} .
$$

Note that the energy density contains the square of the speed of light. Since the mass-charge density is determined by the oscillation frequency, the last formula correlates with the known Einstein's formula for the total energy of a body with a fixed mass.

\section{Biquaternion Representation of Atoms. Simple Gamma}

So, in the biquaternionic representation, the hydrogen atom is a spherical harmonic standing wave with a fixed frequency in the field of EGM chargecurrents.

Since the main characteristic of the hydrogen atom is the oscillation frequency, which determines its mass, on its basis it is possible to construct a periodic system for atoms of substances according to the principle of the musical scale. As the frequency of vibrations increases, the mass of the atom increases.

The musical scale is a system of octaves with frequency doubling for each subsequent octave:

$$
\omega_{H_{0}}, 2 \omega_{H_{0}}, 4 \omega_{H_{0}}, 8 \omega_{H_{0}}, 16 \omega_{H_{0}}, \cdots
$$

The ratio of vibration frequencies for atoms inside the n-octave:

$$
2^{n-1} \omega_{H_{0}}, \cdots, 2^{n} \omega_{H_{0}}
$$

like the ratio of tone frequencies within the musical scale. Number of tones in the musical scale depends on the type of musical system.

There are many musical structures, which are largely related to the national peculiarities of the musical perception of the peoples who created them. Here in Table 1 two musical systems are given ( simple gamma, [7]), which can be taken as a basis, in which the ratio frequency of tones is a rational number. For such tones (notes), there is the total period of oscillations, which is determined by the least common multiple for the period their harmonies, which makes it possible to harmoniously sound the accords from different notes. For each of them, in nature, there are substances that possess the described above properties. Which of them corresponds to Mendeleyev's periodic table? It should be the subject of a special study for specialists in area of physical chemistry, spectral properties of substances.

Perhaps among these three structures in Table 1 there is no such. But a similar musical scale should be, which contains the frequencies of these scales. The number of tones within an octave can be changed with growth octave numbers, but all the similar tones of the previous octave in it should be present, which explains the repeatability of chemical properties of substances in columns of periodic Mendeleyev's system, just as the musical sounds are harmonious for perception for octaves and chords composed of them. 
Table 1. Simple gamma.

\begin{tabular}{cccccccc}
\hline \multicolumn{7}{c}{ Clean structure } \\
\hline Prima (ut) & $\begin{array}{c}\text { Major } \\
\text { secunda } \\
(\mathrm{re})\end{array}$ & $\begin{array}{c}\text { Major } \\
\text { tertius (mi) }\end{array}$ & $\begin{array}{c}\text { Quartus } \\
(\mathrm{fa})\end{array}$ & $\begin{array}{c}\text { Quintus } \\
(\text { sol })\end{array}$ & $\begin{array}{c}\text { Major } \\
\text { sextus (la) }\end{array}$ & $\begin{array}{c}\text { Major } \\
\text { septim (si) }\end{array}$ & Octabas ut \\
\hline$\omega$ & $9 \omega / 8$ & $5 \omega / 4$ & $4 \omega / 3$ & $3 \omega / 2$ & $5 \omega / 3$ & $15 \omega / 8$ & $2 \omega$ \\
\hline Prima & Secunda & Tertius & - & Quintus & Sectus & - & Octabas \\
\hline$\omega$ & $9 \omega / 8$ & $5 \omega / 4$ & - & $3 \omega / 2$ & $5 \omega / 3$ & & $2 \omega$ \\
\hline
\end{tabular}

Proceeding from this, the atoms can be called musical elementary particles with the appropriate names. The hydrogen atom is the note $u t$ of the first natural octave. Correspondingly, the biquaternion of the k-th atom in the $\mathrm{n}$-th octave has the form

$$
\operatorname{Atom}^{n, k}(t, x)=\frac{1}{r} \mathrm{e}^{i w_{n k} t}\left\{-\sin \left(\frac{w_{n k}}{c} r\right)+\left(\cos \left(\frac{w_{n k}}{c} r\right)-\frac{c \sin \left(w_{n k} r / c\right)}{w_{n k} r}\right) e_{x}\right\} .
$$

Here the frequency of oscillations of the atom

$$
w_{n k}=2^{n} \gamma_{k} w_{H_{0}},
$$

where $\gamma_{k}$ is the k-th coefficient in the table of the corresponding musical scale. For it, all the above formulas for a spherical harmonic pulsar are correct by the frequency of oscillations corresponding to it.

\section{Conclusions}

How many such natural octaves exist? Obviously, no less than the number of rows in the periodic system of Mendeleyev.

Let us note that the twelve-tempered musical scale, now accepted in classical music, with twelve notes inside the octave, can not be taken, since the ratio of frequencies of consecutive tones in it is a number irrational $\sqrt[12]{2}$ and the general period of oscillation for any set of tones in the octave does not exist. Complete harmonious sound in this system can not be achieved. This is well known to the orchestral musicians of strings and wind instruments, the sound of which is determined by the above described musical arrangements. As is known, with disproportionate oscillation frequencies, beats occur.

Such periodic systems can be constructed for elementary harmonic leptons (spinors and asymmetric pulsars), whose addition to atoms with the same vibration frequency apparently creates isotopes of these atoms. Moreover, the addition of the spinors is connected with the magnetization of matter. It is possible to construct many different isotopes with the same asymptotic density of the EGM charge. Which of them exist in nature is also a matter of special experimental research.

We also note that this description of atoms is based on the construction of solutions to the equations of the free field of charge-currents. Under the influence 
of external fields, charges and currents are transformed. Their transformation is described by the generalized Dirac equation (see [3]). In particular, under the influence of stationary EGM-fields, the oscillation spectrum shifts, which should be taken into account in the experimental substantiation of the model, are considered here.

At present, the most widespread and canonized representations of light and heavy elementary particles and atoms are constructed on the basis of solutions of the equations of quantum field theory. The bibliography in this direction is half a century old and very extensive. Here we use the names for heavy and light particles adopted in this theory. However, the represented biquaternion model is completely different, deterministic, based on the determination of the real physical characteristics of elementary particles and atoms, rather than probabilistic ones.

\section{References}

[1] Alexeyeva, L.A. (2009) Journal of Physical Mathematics, 1, Article ID: S090604.

[2] Alexeyeva, L.A. (2016) Journal of Modern Physics, 7, 435-444. https://doi.org/10.4236/jmp.2016.75045

[3] Alexeyeva, L.A. (2016) Journal of Modern Physics, 7, 1351-1358. https://doi.org/10.4236/jmp.2016.711121

[4] Alexeyeva, L.A. (2012) Clifford Analysis, Clifford Algebras and their Applications, 7, 19-39.

[5] Abramowitz, M. and Stegun, I., Eds. (1964) Handbook on Mathematical Functions. National Buro of Standards, Applied Mathematical Series, 55.

[6] Vladimirov, V.S. (1978) Generalized Functions in Mathematical Physics. Nauka, Moscow.

[7] Shilov, G.E. (1970) Simple Gamma. Moscow state University Publisher, Brochure $24 \mathrm{p}$. 


\section{Appendix}

To use biquaternions algebra we give here some definitions.

We consider on Minkowski space $\mathbb{M}=\{(\tau, x)\}$ the functional space of biquaternions in hamiltonian form:

$$
\mathbb{B}(\mathbb{M})=\{F=f(\tau, x)+F(\tau, x)\}
$$

$f$ is a complex function, $F=\sum_{j=1}^{3} F_{j} e_{j}-$ a three-dimensional complex vectorfunction; $x=\sum_{j=1}^{3} x_{j} e_{j}, e_{0}=1, e_{1}, e_{2}, e_{3}$ are basic elements.

We assume $f(\tau, x), F_{j}(\tau, x)$ are locally integrable and differentiable on $\mathbb{M}$ or, in general case, they are generalized functions.

Summation and quaternionic multiplication are defined as

$$
\begin{gathered}
\alpha \mathbf{F}+\beta \mathbf{B}=\alpha(f+F)+\beta(b+B) \triangleq(\alpha f+b \beta)+(\alpha F+\beta B), \\
\mathbf{F} \circ \mathbf{B}=(f+F) \circ(b+B) \triangleq f b-(F, B)+f B+b F+[F, B],
\end{gathered}
$$

where $(F, B)=F_{j} B_{j},[F, B]=\epsilon_{j k l} e_{j} F_{k} B_{l}$ are usual scalar and vector productions in $R^{3}$ (here over repeated indexes there are summation from 1 to $3, \epsilon_{j k l}$ is Levi-Civita symbol).

The norm and pseudonorm of Bq. are denoted

$$
\|\mathrm{F}\|=\sqrt{|f|^{2}+\|F\|^{2}}, \quad\langle\langle\mathrm{~F}\rangle\rangle=\sqrt{|f|^{2}-\|F\|^{2}}, \quad|f|^{2}=\overline{f f}, \quad\|F\|^{2}=(F, \bar{F}) .
$$

We'll use convolution of biquaternions:

$$
\mathbf{F} * \mathbf{B}=f * b-\left(F_{j} * B_{j}\right)+f * B_{j} e_{j}+b * F_{j} e_{j}+\epsilon_{j k l} e_{j} F_{k} * B_{l} .
$$

For regular components a convolution has the form:

$$
f * b=\int_{\mathbb{M}} f(\tau-t, x-y) b(t, y) \mathrm{d} t \mathrm{~d} y_{1} \mathrm{~d} y_{2} \mathrm{~d} y_{3},
$$

to take a convolution for singular generalized function and conditions of convolution existence see [6].

Mutual bigradients $\nabla^{+}, \nabla^{-}$are the differential operators

$$
\begin{aligned}
\nabla^{ \pm} \mathbf{B} & =\left(\partial_{\tau} \pm i \nabla\right) \circ(b+B) \triangleq \partial_{\tau} b \mp i(\nabla, B) \pm i \nabla b \pm \partial_{\tau} B \pm i[\nabla, B] \\
& \triangleq \partial_{\tau} b \mp i \operatorname{div} B \pm i \operatorname{grad} b \pm \partial_{\tau} B \pm i \operatorname{rot} B
\end{aligned}
$$

which are taken corresponding to the sign. 